\title{
Estudo fitoquímico das partes aéreas de Aristolochia birostris Ducht. (Aristolochiaceae)
}

\author{
Vanusia C. França ${ }^{*}$, Karlete Vânia M. Vieira ${ }^{2}$ Edeltrudes de O. Lima ${ }^{3}$, José M. Barbosa- \\ Filho $^{3}$, Emidio V.L. da-Cunha ${ }^{2,3}$, Marcelo Sobral da Silva ${ }^{3}$
}

${ }^{1}$ Departamento de Química, Universidade Estadual da Paraíba, CCEN, 58100-000, Campina Grande, PB, Brasil, ${ }^{2}$ Departamento de Farmácia, Universidade Estadual da Paraíba, CCBS, 58100-000, Campina Grande, PB, Brasil, ${ }^{3}$ Laboratório de Tecnologia Farmacêutica "Delby Fernandes de Medeiros", Universidade Federal da Paraíba, Cx. Postal 5009, 58051-970, João Pessoa, PB, Brasil

\begin{abstract}
RESUMO: A família Aristolochiaceae apresenta entre seus constituintes terpenóides, alcalóides e lignóides. Papo-de-peru, jarrinha, mil-homens e capivara são nomes populares da espécie Aristolochia birostris, a qual é utilizada na medicina popular como antiofídica, sudorífica, anticatarral e abortiva. Um estudo fitoquímico anterior com as partes aéreas desta espécie reportou o isolamento de quatro terpenóides, um lignóide e uma antraquinona. Dando continuidade a esse estudo, este trabalho relata a identificação de 4 lignóides, sendo dois do tipo ariloxiarilpropânico (4-metilenodioxi-5,3' 5' -trimetoxi-1'-alil-8.O.4'-neolignana e 3,4,5,3',5'-pentametoxi-1'-alil8.O.4'-neolignana), um do tipo tetraidrofurano (grandisina) e uma lignana dibenzilbutirolactônica [(-)-hinoquinina], além de vanilina. As estruturas foram estabelecidas através de métodos espectroscópicos de infravermelho e ressonância magnética nuclear uni e bidimensionais. Os lignóides foram avaliados quanto a atividade antimicrobiana frente a algumas cepas de bactérias, fungos e leveduras, entretanto foram inativos.
\end{abstract}

Unitermos: Aristolochia birostris, Aristolochiaceae, neolignana, lignana, atividade antimicrobiana.

\begin{abstract}
Phytochemical study of the aerial parts of Aristolochia birostris Ducht. (Aristolochiaceae)". The family Aristolochiaceae presents among its chemical constituents terpenoids, alkaloids and lignoids. Aristolochia birostris is a species of this family popularly known as "papo-de-peru", "jarrinha", "mil-homens" and "capivara", and it is used in the folk medicine to treat snake bites, as a sudorific, expectorant and abortive. A previous phytochemical study of the aerial parts of this plant reported the isolation of four terpenoids, lignoids and antraquinone. Continuing this study it is now reported the isolation of four lignoids, two of the type aryloxyarylpropane (4-methylenedioxy-5,3' 5'-trimethoxy-1'-alyl-8.O.4'-neolignan and 3,4,5,3',5'-pentamethoxy-1'alyl-8.O.4'-neolignan), one tetrahydrofuran (grandisin) and a dibenzylbutyrolactone lignans [(-)hinokinin], and also vanillin. The chemical structures of the isolated compounds were established based on spectral data, mainly IR and NMR including 1D and 2D experiments. The lignoids were evaluated for their activity against some bacteria (Staphylococcus epidermidis, Staphylococcus aureus, Pseudomonas aeruginosa, Enterococcus faecalis and Escherichia coli), fungi (Aspergilus flavus and Microsporum canis) and yeast (Candida albicans, Candida tropicalis and Criptococcus neoformans), but they were inactive.
\end{abstract}

Keywords: Aristolochia birostris, Aristolochiaceae, neolignan, lignan, antimicrobial activity.

\section{INTRODUÇÃO}

A família Aristolochiaceae é constituída por apenas sete gêneros (Apama, Aristolochia, Asarum, Euglypha, Holostylis, Thottea e Saruma) e cerca de 600 espécies, representadas em todas as regiões do globo terrestre, exceto na Ártica e Antártica. A distribuição não é uniforme e privilegia as zonas tropicais. O gênero Asarum, o segundo em número de espécies da família, suporta melhor o clima frio, enquanto Aristolochia é mais afeito ao clima tropical, ocorrendo na Amazônia, Peru, Bolívia e Colômbia, além de Índia e África. Apenas os gêneros Aristolochia, Euglypha e Holostylis são encontrados em território brasileiro (Hoene, 1942; Correa, 1984).

O gênero Aristolochia é composto por 400 espécies (Chan et al., 1999). Cerca de 62 espécies deste gênero ocorrem no Brasil, sendo conhecidas vulgarmente como "mil-homens", "papo-de-peru", "mata-porcos", "patinho", "jarrinha", etc. Os indígenas as denominam "anhangá-potyra", "flor-do-espírito-do-mal", "flor-dodiabo", "urubu-caa" e "erva-do-urubu", devido aos efeitos alucinógenos que apresentam, sendo utilizadas em ponta 
de flechas para o abate de suas caças (Hoene, 1942). O nome Aristolochia (do grego aristos: excelente e lochios: parto) reflete o uso tradicional na medicina popular como emenagoga e para facilitar o parto (Mengue et al., 2001).

A literatura relata que o gênero Aristolochia apresenta entre seus constituintes 275 terpenóides, 57 lignóides, 167 alcalóides e alcamidas e 99 derivados fenólicos (Lopes et al., 2001). Esta diversidade química contribui para diversas atividades farmacológicas.

A ocorrência do gênero Aristolochia no estado da Paraíba está restrito às espécies $A$. birostris Duchtr. e A. papillaris Mast. A espécie A. birostris é uma trepadeira glabra de caule delicado, cujas folhas são longo-pecioladas, flores axilares, pequenas e solitárias, amarelo-pardacentas e lábio em forma de bico curvado, fruto cápsula oblonga e sementes arredondadas. Todas as partes da planta exalam cheiro desagradável, suas raízes são usadas como antiofídico, em especial contra veneno de cascavel (Crotalus durissus), e suas folhas como sudoríficas e anticatarrais (Correa, 1984).

Publicações anteriores sobre Aristolochia birostris descrevem o isolamento, nas raízes, de sete lignóides (Conserva et al., 1990) e, nas partes aéreas, de quatro terpenóides, um lignóide e uma antraquinona (França et al., 2003). Este artigo relata o isolamento de quatro lignóides e um benzaldeído, bem como a avaliação da atividade antimicrobiana desses lignóides frente a bactérias, fungos e leveduras.

\section{MATERIAL E MÉTODOS}

\section{Procedimentos gerais}

Para as colunas cromatográficas (CC) utilizou-se sílica gel da Merck (art. 7734) e para as placas cromatográficas analíticas (CCDA) e preparativas (CCDP) a sílica gel $\mathrm{PF}_{254}$ Merck (art. 7749). Para os sistemas de eluentes foram utilizados hexano e clorofórmio, puros ou em mistura binária, segundo gradiente crescente de polaridade. As revelações das cromatoplacas foram realizadas por irradiação com UV, em comprimentos de onda 254 e $366 \mathrm{~nm}$, em aparelho MINERALIGTH - modelo UVGL-58, e impregnação com vapores de iodo para as cromatoplacas analíticas. As frações foram reunidas de acordo com os Rf's apresentados em CCDA.

Para a identificação das substâncias os espectros de RMN foram registrados em espectrômetro VARIAN-MERCURY, operando a $200 \mathrm{MHz}$ para ${ }^{1} \mathrm{H}$ e a $50 \mathrm{MHz}$ para ${ }^{13} \mathrm{C}$. Na dissolução das amostras utilizouse $\mathrm{CDCl}_{3}$, sendo o sinal do solvente $(7,2 \mathrm{ppm})$ usado como referência. Os deslocamentos químicos $(\delta)$ foram expressos em ppm e as constantes de acoplamento $(J)$ em Hz. Os espectros de absorção na região do infravermelho (IV) foram obtidos em espectrômetro PERKIN-ELMER FT-IR-1750, na faixa de 4000 a $400 \mathrm{~cm}^{-1}$, utilizando pastilhas de $\mathrm{KBr}$.

\section{Material vegetal}

Partes aéreas da planta foram coletadas em março de 2001 no município de Santa Rita-PB. Exsicata (Agra-4295) encontra-se depositada no Herbário Lauro Pires Xavier da Universidade Federal da Paraíba.

\section{Extração e isolamento dos constituintes químicos}

O material vegetal $(7.800 \mathrm{~g})$ foi submetido à secagem em estufa com circulação de ar, à temperatura de $45^{\circ} \mathrm{C}$ durante 3 dias e, após pulverização, forneceu 1.780 g. O material pulverizado foi submetido à extração em etanol a 95\%, a temperatura ambiente. Após filtração e concentração em rotaevaporador o extrato etanólico bruto $(570 \mathrm{~g})$ foi submetido à partição com hexano $(50 \mathrm{~g})$ e com clorofórmio $(20 \mathrm{~g})$. Os extratos obtidos foram, separadamente, fracionados através de técnicas cromatográficas.

A fase hexânica foi submetida a CC, inicialmente, eluida em hexano puro e, posteriormente, em hexano:clorofórmio. As frações 78-95 foram submetidas a novo fracionamento em coluna e levou ao isolamento das substâncias $1(0,075 \mathrm{~g})$ e $\mathbf{2}(0,070 \mathrm{~g})$, além de cerca de $1,700 \mathrm{~g}$ de $\beta$-sitosterol. As frações $96-103$, obtidas da eluição em clorofórmio puro, forneceram a substância 3 $(0,010 \mathrm{~g})$.

A fase clorofórmica foi submetida ao mesmo processamento cromatográfico da fase hexânica. As frações 13-18 foram submetidas a CCDP em hexano: clorofórmio (8:2) e forneceram, novamente, a mesma quantidade da substância 3 , além da substância $4(0,008$ g). As frações 36-76, obtidas da eluição em clorofórmio puro, após CCDP em hexano:clorofórmio (6:4), forneceu a substância $5(0,020 \mathrm{~g})$.

\section{Avaliação da atividade antimicrobiana}

Preparo das substâncias para avaliação microbiológica: solubilizadas em DMSO e uma gota de Tween 80 para obtenção da concentração de $10.000 \mu \mathrm{g} /$ $\mathrm{mL}$. Além das quatro substâncias relatadas neste trabalho testou-se também a neolignana (diidrocarinatina) anteriormente isolada (França et al., 2003). Foram selecionadas cepas de Staphylococcus epidermidis (ATCC 12228), Pseudomonas aeruginosa (LM 23), Staphylococcus aureus (ATCC 6538), Enterococcus faecalis (LM 12), Staphylococcus aureus (ATCC 25923), Escherichia coli (LM 51), Candida albicans (FCF 243), Candida tropicalis (LM 9), Aspergilus flavus (LM 21), Criptococcus neoformans (ICB 59) e Microsporum canis (LM 819). Estes microrganismos foram isolados e identificados no Laboratório de Micologia do Departamento de Ciências Farmacêuticas da Universidade Federal da Paraíba, sendo mantidos a $4^{\circ} \mathrm{C}$ em agar Muller-Hinton (Merck) para bactérias e leveduras e agar Saboraud dextrose (DIFCO) para fungos filamentosos. Os 
testes foram realizados pelo método de difusão em meio sólido, processo cavidade-placa [Vincent; Vincent (1944); Mc Ginnis (1980); Odds (1989); Mimn et al. (1995)]. Nos experimentos, as cepas foram incubadas a $37^{\circ} \mathrm{C}$, durante 48 horas (bactérias e leveduras) e a temperatura ambiente por 7-14 dias (fungos filamentosos). O controle negativo do microrganismo foi feito semeando-o em meio isento de antimicrobiano, verificando o seu crescimento, enquanto o positivo efetuou-se por meio das drogas padrões, tetraciclina $(30 \mu \mathrm{g} / \mathrm{mL})$ para bactérias e cetoconazol $(200 \mu \mathrm{g} / \mathrm{mL})$ para fungos. Os testes foram realizados em duplicata e o resultado final foi determinado pela média dos halos de inibição, sendo considerados positivos aqueles com diâmetro igual ou superior a $9 \mathrm{~mm}$.

\section{RESULTADOS E DISCUSSÃO}

As substâncias 1 e 2 apresentaram-se como óleo amarelado e os respectivos espectros de $\mathrm{RMN}{ }^{13} \mathrm{C}$ evidenciaram deslocamentos para carbonos metilênicos $\left(\delta_{\mathrm{C}} 40,2\right.$ e $\left.\delta_{\mathrm{C}} 115,5\right)$ e de carbono metínico $\left(\delta_{\mathrm{C}} 137,9\right)$ característicos de grupo alila. Sinais de carbonos metínicos na região de carbonos aromáticos foram compatíveis com anel tetra-substituído. Esqueleto ariloxiarilpropânico foi proposto para ambas as substâncias a partir dos sinais dos carbonos metílico, metilênico e oximetínico (Tabela 1). $\mathrm{O}$ que as diferenciou foram os sinais para um grupo metilenodioxi $\left(\delta_{\mathrm{C}} 101,1\right)$ em 1 e para grupo metoxila $\left(\delta_{\mathrm{C}} 60,9\right)$ impedida estericamente em 2 (Tabela 1). Os espectros de $\mathrm{RMN}{ }^{1} \mathrm{H}$ para $\mathbf{1}$ e 2 apresentaram sinais característicos de grupo alila $\left[\delta_{\mathrm{H}} 5,98(d d t, J=17,0\right.$; 10,2 e $\left.6,8 \mathrm{~Hz}, 1 \mathrm{H}, \mathrm{H}-8^{\prime}\right), \delta_{\mathrm{H}} 5,10(d d t, J=10,2 ; 2,0$ e
$1,6 \mathrm{~Hz}, 1 \mathrm{H}, \mathrm{H}-9)$ ) $\delta_{\mathrm{H}} 5,05$ (ddt, $J=17,0 ; 2,0$ e $1,6 \mathrm{~Hz}$, $1 \mathrm{H}, \mathrm{H}-9$ ') e $\left.\delta_{\mathrm{H}} 3,31\left(d, J=6,8 \mathrm{~Hz}, 2 \mathrm{H}, \mathrm{H}-7^{\prime}\right)\right]$ e mostrou para 1 a presença de singleto em $\delta_{\mathrm{H}}$ 5,89 integrando para 2 hidrogênios, confirmando a presença de grupo metilenodióxido. Observou-se ainda sinais de duplo dubleto em $\delta_{\mathrm{H}} 3,11(J=13,4$ e $5,2 \mathrm{~Hz}, 1 \mathrm{H})$ e $\delta_{\mathrm{H}} 2,71(\mathrm{~J}=$ $13,4$ e $8,0 \mathrm{~Hz}, 1 \mathrm{H})$ e de multipleto em $\delta_{\mathrm{H}} 4,30$, atribuídos aos hidrogênios benzílico e oximetínico, respectivamente. Os espectros de correlações heteronuclear (HETCOR e HMBC) e homonuclear (COSY e NOESY) confirmaram as atribuições previamente estabelecidas (Silva et al., 1989) para 3,4-metilenodioxi-5,3' 5'-trimetoxi-1'-alil8.O.4'-neolignana (1) e para 3,4,5,3',5'-pentametoxi-1'alil-8.O.4'-neolignana (2) (Braga et al., 1984) (Figura 1). Estes lignóides já foram isolados nas raízes desta espécie (Conserva et al., 1990).

A substância 3 apresentou-se como óleo amarelado com $[\alpha]^{25}-97,8{ }^{0}\left(0,02\right.$ em $\left.\mathrm{CHCl}_{3}\right)$. O espectro de $\mathrm{RMN}{ }^{13} \mathrm{C}$ mostrou deslocamentos químicos para carbono metilênico $\left(\delta_{\mathrm{C}} 101,0\right)$ característico de grupo metilenodioxi e as absorções de carbonos metínicos $\left(\delta_{\mathrm{C}}\right.$ $108,2,108,3,108,7,109,4,121,5$ e 122,2) sugeriram a presença de dois grupos arila tri-substituídos. Sinais de carbono não hidrogenado em $\delta_{\mathrm{C}} 178,3$, característico de carbonila lactônica em anel de cinco membros, e de carbono oximetilênico em $\delta_{\mathrm{C}} 71,1$, foram compatíveis com esqueleto dibenzilbutirolactônico de neolignanas (Tabela 1). O espectro de $\mathrm{RMN}{ }^{1} \mathrm{H}$ mostrou na região de aromáticos dubletos em $\delta_{\mathrm{H}} 6,42(J=8,0 \mathrm{~Hz}), \delta_{\mathrm{H}} 6,60(J$ $=1,6 \mathrm{~Hz}), \delta_{\mathrm{H}} 6,68(J=8,2 \mathrm{~Hz})$ e em $\delta_{\mathrm{H}} 6,70(J=1,6$ $\mathrm{Hz}$ ) atribuídos a $\mathrm{H}-5$ ', $\mathrm{H}-2$ ', $\mathrm{H}-5$ e $\mathrm{H}-2$, respectivamente. Sinais de duplo dubletos em $\delta_{\mathrm{H}} 6,58(J=8,0$ e $1,6 \mathrm{~Hz})$ e<smiles>C=CCc1cc(OC)c(OC(C)Cc2cc(OC)c3c(c2)OCO3)c(OC)c1</smiles><smiles>C=CCc1cc(OC)c(OC(C)Cc2cc(OC)c(OC)c(OC)c2)c(OC)c1</smiles><smiles>O=C1OC[C@H](Cc2ccc3c(c2)OCO3)[C@@H]1Cc1ccc2c(c1)OCO2</smiles><smiles>COc1cc(C=O)ccc1O</smiles>

4<smiles>COc1cc([C@H]2O[C@H](c3cc(OC)c(OC)c(OC)c3)C(C)C2C)cc(OC)c1OC</smiles>

5

3

Figura 1. Substâncias isoladas das partes aéreas. 
Tabela 1. Dados de $\mathrm{RMN}{ }^{13} \mathrm{C}\left(50 \mathrm{MHz}, \mathrm{CHCl}_{3}, \delta\right)$ para os lignóides isolados.

\begin{tabular}{|c|c|c|c|c|c|c|}
\hline $\mathrm{C}$ & 1 & 2 & 3 & 4 & $\mathrm{C}$ & 5 \\
\hline 1 & 133.3 & 134.8 & 131.5 & 129.9 & 1,1 & 137.8 \\
\hline 2 & 103.4 & 106.4 & 108.2 & 108.7 & 2,2 & 103.3 \\
\hline 3 & 148.5 & 153.3 & 147.8 & 147.1 & 3,3 & 153.1 \\
\hline 4 & 135.4 & 136.5 & 146.3 & 151.7 & 4,4 & 137.8 \\
\hline 5 & 143.2 & 153.5 & 108.3 & 114.4 & 5,5 & 153.1 \\
\hline 6 & 108.6 & 106.4 & 121.5 & 127.5 & 6,6 & 103.3 \\
\hline 7 & 43.2 & 43.7 & 34.8 & 190.9 & 2,5 & 87.4 \\
\hline 8 & 79.7 & 79.7 & 46.5 & & 3,4 & 44.3 \\
\hline 9 & 19.5 & 19.8 & 178.3 & & $\mathrm{Me} \mathrm{3,} 4$ & 13.2 \\
\hline 1 & 133.5 & 134.3 & 131.2 & & & \\
\hline 2 & 105.4 & 105.5 & 109.4 & & & \\
\hline 3 & 153.5 & 152.7 & 147.8 & & & \\
\hline 4 & 134.1 & 135.4 & 146.4 & & & \\
\hline 5 & 153.5 & 152.7 & 108.7 & & & \\
\hline 6 & 105.4 & 105.5 & 122.2 & & & \\
\hline $7^{\prime}$ & 40.5 & 40.5 & 38.3 & & & \\
\hline 8 & 137.2 & 137.2 & 41.3 & & & \\
\hline 9 & 115.9 & 115.9 & 71.1 & & & \\
\hline $\mathrm{MeO}-3$ & & 56.1 & & 56.1 & & \\
\hline $\mathrm{MeO}-4$ & & 60.9 & & & & \\
\hline MeO-5 & 56.5 & 56.1 & & & & \\
\hline $\mathrm{MeO}-3,5$ & 55.9 & 56.0 & & & & \\
\hline $\mathrm{MeO}-4,4 "$ & & & & & & 60.9 \\
\hline $\mathrm{MeO}-3,5,3,5^{\prime \prime}$ & & & & & & 56.1 \\
\hline $\mathrm{CH}_{2} \mathrm{O}_{2}$ & 101.1 & & 101.0 & & & \\
\hline
\end{tabular}

em $\delta_{\mathrm{H}} 6,45(J=8,2$ e 1,6 Hz) foram atribuídos a H-6’ e H6 , respectivamente. A presença de grupos metilenodioxi foi confirmada pelos singletos em $\delta_{\mathrm{H}} 5,92$ e $\delta_{\mathrm{H}} 5,91$. Para este tipo de estrutura existem dois centros estereogênicos (C-8 e C-8'), logo configurações relativas cis e trans são possíveis (Lopes, 1983). Quando os hidrogênios H-8 e H-8' são cis os hidrogênios carbinólicos (H-9') tornamse equivalentes e apresentam-se no espectro de $\mathrm{RMN}{ }^{1} \mathrm{H}$ como singleto largo em $\delta_{\mathrm{H}} 4,10$, ao contrário, quando trans esses hidrogênios (H-9') não são equivalentes e absorvem como multipleto em $\delta_{\mathrm{C}} 3,90$. Deste modo a substância 3 foi identificada como hinoquinina (Figura 1). A literatura (Agrawal; Thakur, 1985) relata que diferenças de valores de deslocamentos podem ser observados entre os carbonos dependendo da sua rotação específica $(+)$ ou $(-)$. Assim, através da atividade ótica, pode-se definir 3 como sendo a $8 R, \quad 8 ' R-3,4,3^{\prime}, 4^{\prime}$-dimetilenodioxi-9-oxi-8.8', 9.0.9'lignana, anteriormente isolada nas raízes desta espécie (Conserva et al., 1990).

A substância 4 apresentou-se como cristais branco e o espectro de $\mathrm{RMN}{ }^{13} \mathrm{C}$ registrou apenas 8 sinais, sendo que deslocamentos para carbono metínico $\left(\delta_{\mathrm{C}}\right.$ 190,9), característico de carbonila de aldeído, carbonos não hidrogenados $\left(\delta_{\mathrm{C}} 129,9,147,1\right.$ e 151,7$)$ e para carbonos metínicos $\left(\delta_{\mathrm{C}} 108,7,114,4\right.$ e 127,5) sugeriram anel benzênico tri-substituído (Tabela 1). O espectro de RMN ${ }^{1} \mathrm{H}$ mostrou singletos em $\delta_{\mathrm{H}} 9,81$, confirmando presença de grupo aldeido, e em $\delta_{\mathrm{H}} 3,96$, relativo a grupo metoxilíco. Na região de aromáticos foram observados dubletos em $\delta_{\mathrm{H}} 7,00(J=8,4 \mathrm{~Hz}, \mathrm{H}-5,1 \mathrm{H})$ e $\delta_{\mathrm{H}} 7,38(\mathrm{~J}=$
$1,6 \mathrm{~Hz}, \mathrm{H}-2,1 \mathrm{H})$ e de duplo dubleto em $\delta_{\mathrm{H}} 7,39(J=8,4 \mathrm{e}$ $1,6 \mathrm{~Hz}, \mathrm{H}-6,1 \mathrm{H})$. A posição do grupo metoxi foi definida pelo espectro NOESY, pela correlação deste com H-2. Deste modo foi identificada como sendo 3-metoxi-4hidroxi-benzaldeído, conhecida como vanilina e já isolada anteriormente na família em Aristolochia asclepiadifolia (Lopes et al., 2001) e A. pubescens (Nascimento; Lopes, 1999), entretanto é a primeira vez que está sendo isolada nesta espécie.

A substância 5 apresentou-se como óleo amarelado e o espectro de $\mathrm{RMN}{ }^{13} \mathrm{C}$ mostrou apenas 8 sinais. Deslocamentos químicos para carbono metínico $\left(\delta_{\mathrm{C}} 44,3\right)$, de carbono oximetínico $\left(\delta_{\mathrm{C}} 87,4\right)$ e de carbono metílico $\left(\delta_{\mathrm{C}} 13,2\right)$ sugeriram esqueleto do tipo 2,3diariltetraidrofurânico. Sinais para carbonos oximetílicos $\left(\delta_{\mathrm{C}} 56,1\right.$ e $\left.\delta_{\mathrm{C}} 60,9\right)$ indicaram a presença de grupos metoxi. No espectro de $\mathrm{RMN}{ }^{1} \mathrm{H}$ foi observado um único singleto na região de hidrogênios ligados a carbonos aromáticos $\left(\delta_{\mathrm{H}} 6,64\right)$ atribuído a H-2', H-2", H-6', H-6". Este espectro mostrou dubleto em $\delta_{\mathrm{H}} 4,51(J=6,4 \mathrm{~Hz}, 2 \mathrm{H})$ atribuído a H-2 e H-5, multipleto em $\delta_{\mathrm{H}} 2,32$ atribuído a H-3 e H-4 e outro dubleto em $\delta_{\mathrm{H}} 1,06(\mathrm{~J}=6,6 \mathrm{~Hz}, 6 \mathrm{H})$ relativo a grupos metílicos (Tabela 1). Identificou-se esta substância como sendo a neolignana 2,5-bis-(3',4',5'-trimetoxifenil)-3,4dimetiltetraidrofurano, de nome grandisina, anteriormente isolada nas raízes desta espécie (Conserva et al., 1990).

A avaliação microbiológica mostrou que as substâncias não apresentaram atividade frente as cepas de bactérias, fungos e leveduras utilizadas neste experimento. Devido ao resultado negativo obtido não se 
realizou ensaios para cálculo da Concentração Inibitória Mínima (CIM).

\section{REFERÊNCIAS}

Agrawal PK, Thakur RS 1985. ${ }^{13} \mathrm{C}$ NMR spectroscopy of lignan and neolignan derivatives. Mag Res Chem 23: 299-418.

Braga $\mathrm{ACH}$, Zacchino S, Badano H, Sierra MG, Rúveda EA 1984. ${ }^{13} \mathrm{C}$ NMR spectral and conformational analysis of 8-O-4 neolignans. Phytochemistry 23: 2025-2028.

Chan YY, Leu YL, Wu TS 1999. The constituents of the leaves of Aristolochia heterophylla Hemsl. Chem Pharm Bull 47: 887-889.

Conserva LM, Silva MS, Braz Filho R 1990. Lignans from Aristolochia birostris. Phytochemistry 29: 257-260.

Correa, MP 1984. Dicionário de plantas úteis do Brasil e exóticas cultivadas. Rio de Janeiro: IBDF, volumes I, IV e $\mathrm{V}$.

França VC, Agra MF, Barbosa-Filho JM, Da-Cunha EVL, Silva MS 2003. Physcion and dihydrocarinatin from Aristolochia birostris. Biochem Syst Ecol 31: 13411343.

Hoene FC 1942. Flora Brasílica. São Paulo: Graphicars.

Lopes LMX, Nascimento IR, Silva T 2001. Phytochemistry of the Aristolochiaceae family. In: Research Advances in Phytochemistry, ed.Kerala: Global Research Network, v.2, p. 19-108.

Lopes LMX 1983. Constituintes químicos de frutos de Virola sebifera. São Paulo vol. I, 135p. Tese de Doutorado - Universidade de São Paulo.

Mc Ginnis, MR 1980. Laboratory handbook of medical mycology. New York: Academic Press.

Mengue SS, Mentz LA, Schenkel EP 2001. Uso de plantas medicinais na gravidez. Rev Bras Farmacogn 11: 21-35.

Mimn CA, Willians D, Pfaifer J 1995. Microbiologia médica. São Paulo: Editora Manole.

Nascimento IR, Lopes LMX 1999. 2,3-Dihydrobenzofuran neolignans from Aristolochia pubescens. Phytochemistry 52: 345-350.

Odds FC 1989. Antifungal activity of saperconazole (R. 66905) in vitro. J Antimicrobial Chemother 24: 533-537.

Silva MS, Barbosa-Filho JM, Yoshida M, Gottlieb OR 1989. Benzodioxane and $\beta$-aryloxy-arylpropane type neolignans from Licaria chrysophylla. Phytochemistry 28: 3477-3482.

Vincent JG, Vincent HM 1944. Filter paper disc modification of the Oxford cup penicillin determination. Proc Soc Biol Med 25: 162-164. 